\title{
THE UNITED STATES VERSION OF THE STROKE DRIVERS' SCREENING ASSESSMENT BATTERY: A REPORT OF PRELIMINARY FINDINGS
}

\author{
Abiodun Emmanuel Akinwuntan ${ }^{1}$, Diana Gantt ${ }^{1}$, Gina Gibson ${ }^{1}$, Kurt Kimmons ${ }^{1}$, \\ Valerie Ross ${ }^{1}$, Peter Rosen ${ }^{2,3}$, \& Jerry Wachtel ${ }^{4}$ \\ ${ }^{1}$ Georgia Health Sciences University, Augusta, Georgia \\ ${ }^{2}$ Sharp Rees-Stealy Medical Group \\ ${ }^{3}$ Department of Ophthalmology, University of California, San Diego \\ San Diego, California, USA \\ ${ }^{4}$ The Veridian Group, Inc. \\ Berkeley, California, USA \\ E-mail: aakinwuntan@georgiahealth.edu
}

\begin{abstract}
Summary: We investigated the potential for predicting driving performance of a United States (US)-based population of participants using an adapted version of the Stroke Drivers' Screening Assessment (SDSA) battery. Participants included seven first-ever stroke survivors (age $51 \pm 8$ years) and 11 individuals with Hoehn \& Yahr Stage 2 or 3 Parkinson's disease (PD) (age 65 \pm 8 years). We adapted the original United Kingdom (UK) version of the SDSA to make it suitable for use in the US by replacing all UK-specific traffic situations and road signs with their US equivalents. Following administration of the adapted (US) version of the SDSA, stroke participants' driving performance was evaluated in a driving simulator. PD participants' driving performance was evaluated in the driving simulator as well as on-road. The pass/fail SDSA equations of the original UK version of the SDSA were applied to predict the pass/fail outcome of participants' driving evaluation. The SDSA predicted stroke participants' simulator-based driving performance with 100\% accuracy. The SDSA predicted PD participants' simulator-based performance with 73\% accuracy and the on-road performance with 82\% accuracy. The accuracy with which driving performance of stroke and PD participants in this preliminary study was predicted by the US version of the SDSA is promising and informs the need for a larger study to better investigate and validate its predictive accuracy.
\end{abstract}

\section{INTRODUCTION}

Driving is one of the very important activities of daily living (ADL) affected by the motor, visual, cognitive and perceptual deficits commonly experienced after stroke (Akinwuntan et al, 2002). Between $30 \%$ and $43 \%$ of the approximately six million stroke survivors in the US resume driving within one year of the stroke incidence. However, more than $87 \%$ of these individuals resume driving without any formal evaluation of their driving ability (Fisk et al, 1997). Reasons for the low rate of evaluation are the absence of standard practices, the lack of generally accepted and valid screening tools, the duration, and cost of current evaluation practices. The SDSA battery, which was developed in the United Kingdom (UK), consists of four neuropsychological tests that together take 28 minutes to administer (Nouri \& Lincoln, 1992). The battery has been reported as predictive of the driving performance of stroke survivors (Nouri \& Lincoln, 1993, Devos et al, 2010), and its validity in measuring attention and executive 
abilities critical for driving has been demonstrated (George \& Crotty, 2010). The SDSA has been adapted for use in Nordic countries with moderate (62\%) (Selander et al, 2010) to moderately high accuracy (78\%) (Lundberg et al, 2003) in predicting stroke survivors' driving performance. Component tests of the SDSA also combined with other cognitive measures to predict driving after Parkinson's disease (PD) (Radford et al, 2004), multiple sclerosis (Lincoln \& Radford, 2008), dementia (Lincoln et al, 2006), and traumatic brain injury (Radford et al, 2004). To date, however, the SDSA battery has not been used in the US because two of its tests incorporate traffic rules and road signs that are different from those used in the US.

In light of the usefulness of the SDSA in predicting on-road performance after stroke and other neurological conditions, we adapted the SDSA battery to make it suitable for use in the US. The aim in this study was to investigate the potential for predicting driving performance of a USbased cohort of stroke survivors and individuals with PD using performance on the SDSA. Based on the reported accuracies of the UK version of the SDSA, 83\% in Nouri \& Lincoln, 1992 and $81 \%$ in Nouri \& Lincoln, 1993, in predicting on-road performance of stroke survivors, we hypothesized that the US version of the SDSA would predict participants' driving performance with at least $80 \%$ accuracy.

\section{METHODS}

\section{Participants}

Participants were recruited through use of flyers, newspaper advertisements, and word of mouth in the Augusta, Georgia area, as well as referrals from local neurologists. Participants' informed consent was obtained through the use of a document approved by the Georgia Health Sciences University (GHSU) Human Assurance Committee. To be included, participants had to have a diagnosis of first-ever stoke based on CT/MRI evidence or Stage 2 or 3 PD using the Hoehn \& Yahr Scale. GHSU neurologists verified participants’ diagnosis. Participants also had to possess a valid drivers license, drove at least 10 miles per week pre- stroke or currently drive at least 10 miles per week (PD participants), had binocular visual acuity of better than or equal to 20/60 with or without correction and were ambulatory with or without an assistive device. Exclusion criteria were a history of multiple sclerosis, diagnosis of Alzheimer's disease, psychiatric disorder, an epileptic seizure within the previous year, and anopia in any field of view. Each participant completed a pretesting questionnaire to document demographic and driving information such as age, gender, level of education, driving experience, physical functional status (Barthel Index) and cognitive status (Mini-Mental State Examination).

\section{Adaptation of the SDSA}

The SDSA contains four component tests: Dot Cancellation Test (DCT), Square Matrix Direction (SMD), Square Matrix Compass (SMC), and Road Sign Recognition (RSR). Briefly, in the DCT, participants are instructed to cross out all groups of 4 dots on a paper containing 625 groups of 3, 4, and 5 dots in a maximum time of 15 minutes. The duration (time), number of uncrossed groups of 4 dots (errors), and number of crossed groups of 3 or 5 dots (false positives) are documented. The SMD and SMC each involve correctly placing cards, each of which contains pictures of 2 vehicles traveling in different directions, into 16 squares arranged in a 4 by 
4 matrix in a maximum of 5 minutes. A correctly placed card earns 2 points, and the range maximum score in each test is 32. The RSR involves matching the best road sign from a selection of 19 road signs to 12 traffic situations in a maximum time of 3 minutes. One point is awarded for each correct match. A full description of the SDSA and how each of the tests is administered and scored can be found in several sources (e.g. Nouri \& Lincoln, 1992, Lundberg et al. 2003). To make the SDSA suitable for use with a US-based cohort, the SMD and RSR tests were adapted to US traffic conditions. In the SMD test, the position of the driver behind the wheel was changed from right to left (Figure 1a).

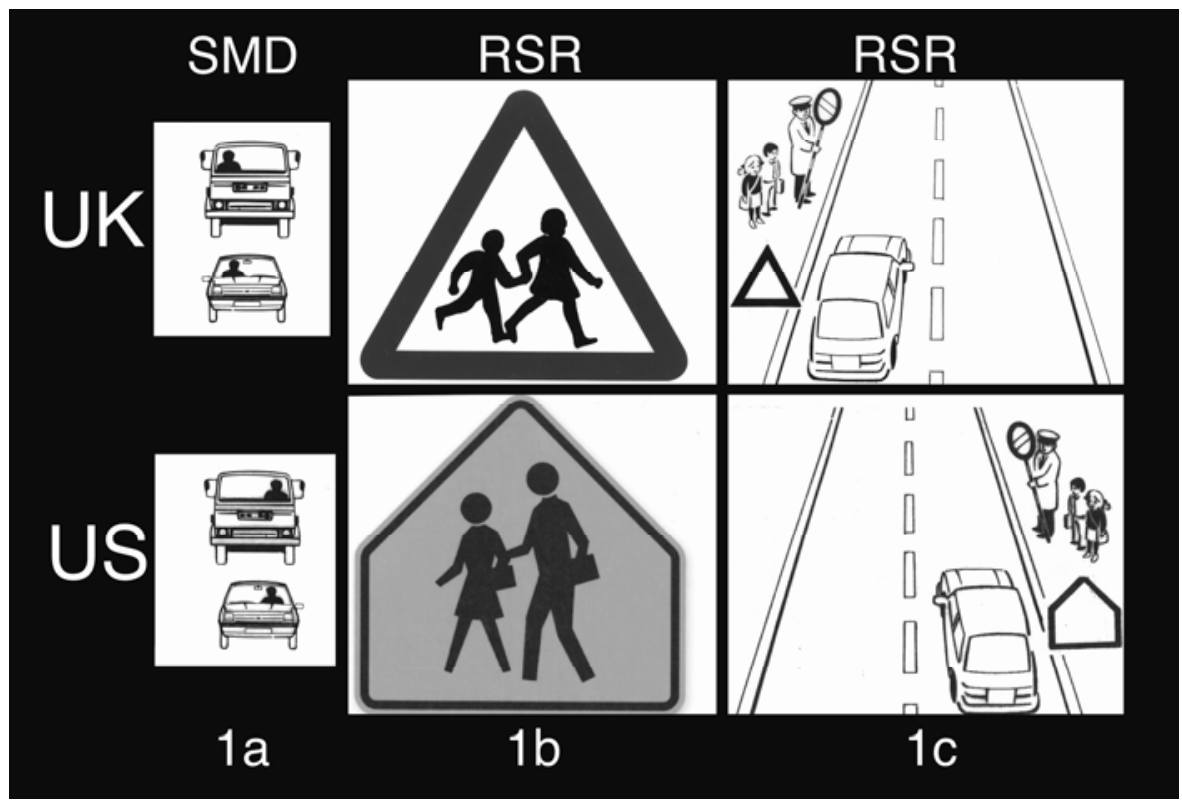

Figure 1. Examples of modifications to the UK version of the SDSA

In the RSR test, all road signs and traffic situations were changed. Examples of a UK road sign and its US equivalent are shown in Figure $1 \mathrm{~b}$ and a UK traffic situation and its US equivalent in Figure 1c. All changes were done in consultation with one of the developers of the original SDSA, experts knowledgeable about the US Manual of Uniform Traffic Control Devices (Federal Highway Administration, US Department of Transportation, 2009) and driving assessment specialists. Prior to implementation, the modified (US) version of the SDSA was pilot tested with 10 US-based healthy participants.

\section{Simulator-based evaluation of driving performance}

Participants' driving performance was evaluated in a driving simulator. To familiarize them with simulator-based driving, participants first drove through a 2-mile scenario containing simple traffic events without being evaluated. Driving performance was then evaluated while the participants drove through a simulated 9.5-mile course under traffic conditions similar to those of a regular 10.30 a.m. work day in the Augusta, GA area. The 9.5-mile course contained straight, slightly curved or hilly 2-lane (rural), 4-lane (urban), and 6-lane (highway) roads. All roadways had posted speed limits between 30 and $70 \mathrm{mph}$, standard lane markings, and intersections with and without traffic signals as well as human and vehicular interactions. Prior to beginning the drive, participants were instructed to drive as in real-life and to obey all traffic rules. Participants 
drove another 1.5-mile course during which reaction time was evaluated.This course contained only a straight 2-lane road (one lane in each direction) with a $45 \mathrm{mph}$ speed limit. At five predetermined times, unknown to the participants, a large 'STOP' sign appeared in the center of the scene. Participants were instructed to maintan an average speed of $45 \mathrm{mph}$ and to react as quickly as possible whenever a 'STOP' sign appeared by bringing the car to a complete stop and resume driving immediately thereafter. All events in the 2-mile familiarization, 9.5-mile, and 1.5-mile evaluation scenarios were developed using the Scenario Definition Language (SDL) of the STISIM Drive System Model 400. The simulator was built into the body of a 1991 Plymouth Acclaim automobile. The scenarios were projected with a $135^{\circ}$ horizontal field of view on three 9’ x 9' screens placed directly in front of the car.

Performance parameters during the 9.5-mile simulated drive were automatically generated by the simulator software. The parameters included time-to-collision (TTC), number of road edge excursions, centerline crossings, speed limit violations, collisions, pedestrians hit, and runtime. In the 1.5-mile scenario, simple reaction time was calculated as the "time from presentation of each 'STOP'sign to the time of significant decrease in the throttle force," and complex reaction time was calculated as the "time from presentation of each 'STOP' sign to the time when the car came to a complete stop (i.e. speed = zero)”. An algorithm derived from age-based normative values of all the simulator parameters (Akinwuntan et al, 2009) was used to classify participants' performance as 'pass' or 'fail'. Briefly, values below the average age-based normative value for each parameter were assigned no points, values between the average and maximum values were assigned 1 point, and values above the maximum values were assigned 2 points. A summed score of less than 5 was classified as "pass" and 5 or more points as "fail".

\section{On-road evaluation of driving performance}

Only participants with PD ( $\mathrm{n}=11)$ had their driving performance further evaluated during an actual on-road test due to lack of funding to support the on-road evaluation of our stroke participants. The on-road driving evaluation was conducted in a vehicle adapted for safety and registered with the Georgia Department of Driver Services. The evaluation was administered by a driving assessment expert with over 33 years experience in older-driver education and evaluation of on-road driving abilities. The on-road evaluation took place on a standardized road course traversing rural (light traffic), urban (moderate to heavy traffic), and interstate highways. The road network included multiple intersections with and without traffic-signals, 4-way stop signs, and several other traffic and road signs. At the end of the on-road evaluation, the driving assessment expert made a "pass" or "fail" decision using scores he entered on a 10-item check list during the evaluation.

\section{Data analysis}

Stroke and PD participants’ demographic, physical and cognitive characteristics were compared using t-tests or Fisher's exact tests. We used the pass/fail equations developed for the original SDSA (UK version) to predict outcomes of the simulator and on-road driving evaluations. In addition, we compared the outcome of PD participants' simulator evaluation with the outcome of their on-road evaluation to determine the similarity between the two evaluation methods. 


\section{RESULTS}

Our PD participants were significantly older, had more years of driving experience, and were more cognitively impaired when compared with the stroke participants (Table 1).

Table 1. Comparison of stroke and PD participants characteristics

\begin{tabular}{lcccc}
\hline Variables & Stroke $(\mathrm{n}=7)$ & $\mathrm{PD}(\mathrm{n}=11)$ & Statistic & Significance \\
\hline Age, yrs & $51 \pm 8$ & $65 \pm 8$ & $\mathrm{t}=-3.73$ & 0.0018 \\
Gender, Male/Female & $4 / 3$ & $8 / 3$ & $\mathrm{X}_{\mathrm{F}}=0.31$ & 0.6267 \\
Education, High school/College/Graduate & $3 / 3 / 1$ & $4 / 4 / 3$ & $\mathrm{X}_{\mathrm{F}}=0.15$ & 1.000 \\
Drive Experience, yrs & $35 \pm 5$ & $49 \pm 7$ & $\mathrm{t}=-4.50$ & 0.0004 \\
Barthel Index, /100 & $87 \pm 17$ & $95 \pm 5$ & $\mathrm{t}=-1.65$ & 0.1186 \\
Mini Mental State Examination, /30 & $28 \pm 2$ & $24 \pm 5$ & $\mathrm{t}=2.52$ & 0.0227 \\
\hline
\end{tabular}

$\mathrm{PD}=$ Parkinson's Disease; $\mathrm{t}=\mathrm{t}$-test; $\mathrm{X}_{\mathrm{F}}=$ Fisher's Exact

The pass (1) and fail (2) equations of the original SDSA are:

$$
\begin{aligned}
& \left(\text { DCT time } * 0.012+\text { DCT false positive } * 0.216+S M D * 0.409+R S R^{*} 1.168\right)-13.79 \\
& (\text { DCT time*0.017 + DCT false positive*0.035 +SMD*0.185 + RSR*0.813) }-10.042
\end{aligned}
$$

When the "Pass" score of a participant is higher than the "Fail" score, that individual is predicted to pass, and vice versa. The equations accurately predicted all seven stroke participants' simulator-based pass/fail performance (Table 2a). The equations predicted PD particpants' simulator-based performance with 73\% accuracy (sensitivity $=80 \%$, specificity $=67 \%$ ) $($ Table b) and their on-road driving performance with $82 \%$ accuracy (sensitivity $=100 \%$, specificity $=$ 71\%) (Table 2c).

Table 2. Results of all the predictions

(a) Stroke participants' SDSA vs. simulator-

\begin{tabular}{|c|c|c|}
\hline \multirow{4}{*}{$\begin{array}{l}\text { SDSA pass/fail } \\
\text { Fail, } \mathrm{n}(\%) \\
\text { Pass, } \mathrm{n}(\%)\end{array}$} & \multicolumn{2}{|c|}{ Simulator-based pass/fail } \\
\hline & Fail, n(\%) & Pass, n(\%) \\
\hline & $\mathrm{a}=4(57.1)$ & $\mathrm{b}=0(0.0)$ \\
\hline & $c=0(0.0)$ & $\mathrm{d}=3(42.9)$ \\
\hline
\end{tabular}
based performance $(n=7)$

(c) PD participants' SDSA vs. on-road

\begin{tabular}{|c|c|c|}
\hline \multirow[b]{2}{*}{$\begin{array}{l}\text { SDSA pass/fail } \\
\text { Fail, n(\%) } \\
\text { Pass, n(\%) }\end{array}$} & \multicolumn{2}{|c|}{ On-road pass/fail } \\
\hline & $\begin{array}{l}\text { Fail, } \mathrm{n}(\%) \\
\mathrm{a}=4(36.4) \\
\mathrm{c}=0(0.0)\end{array}$ & $\begin{array}{l}\text { Pass, } n(\%) \\
b=2(18.2) \\
d=5(45.4)\end{array}$ \\
\hline
\end{tabular}
performance $(\mathrm{n}=11)$ (b) PD participants’ SDSA vs. simulator-based performance $(\mathrm{n}=11)$

\begin{tabular}{cll}
\hline & \multicolumn{2}{c}{ Simulator-based pass/fail } \\
\cline { 2 - 3 } SDSA pass/fail & Fail, $\mathrm{n}(\%)$ & Pass, $\mathrm{n}(\%)$ \\
Fail, $\mathrm{n}(\%)$ & $\mathrm{a}=4(36.4)$ & $\mathrm{b}=2(18.2)$ \\
Pass, $\mathrm{n}(\%)$ & $\mathrm{c}=1(09.1)$ & $\mathrm{d}=4(36.4)$ \\
\hline
\end{tabular}

(d) PD participants' simulator-based vs. on-road performance $(\mathrm{n}=11)$

\begin{tabular}{clc}
\hline & \multicolumn{2}{c}{ On-road pass/fail } \\
\cline { 2 - 3 } Simulator pass/fail & Fail, $\mathrm{n}(\%)$ & Pass, $\mathrm{n}(\%)$ \\
Fail, $\mathrm{n}(\%)$ & $\mathrm{a}=4(36.4)$ & $\mathrm{b}=1(09.1)$ \\
Pass, $\mathrm{n}(\%)$ & $\mathrm{c}=0(0.0)$ & $\mathrm{d}=6(54.5)$ \\
\hline
\end{tabular}

Although two PD participants whom we had predicted to fail, in fact passed the on-road evaluation (positive predictive value $=67 \%$ ), all five whom we had predicted to pass, in fact passed the on-road evaluation (negative predictive value $=100 \%$ ). As shown in Table $2 \mathrm{~d}$, the simulator-based driving evaluation predicted PD participants' on-road evaluation outcome with 
91\% accuracy, $100 \%$ sensitivity, and $86 \%$ specificity. The positive and negative predictive values of the prediction were $80 \%$ and $100 \%$ respectively.

Table 4. PD participants' SDSA score versus on-road driving performance $(n=11)$

\begin{tabular}{ccc}
\hline & \multicolumn{2}{c}{ On-road driving performance } \\
\cline { 2 - 3 } SDSA pass/fail & Fail, $\mathrm{n}(\%)$ & Pass, $\mathrm{n}(\%)$ \\
Fail, $\mathrm{n}(\%)$ & $\mathrm{a}=4(36.4)$ & $\mathrm{b}=2(18.2)$ \\
Pass, $\mathrm{n}(\%)$ & $\mathrm{c}=0(0.0)$ & $\mathrm{d}=5(45.4)$ \\
\hline
\end{tabular}

Table 5. PD participants' simulator-based versus on-road driving performance $(n=11)$

\begin{tabular}{|c|c|c|}
\hline \multirow{3}{*}{$\begin{array}{l}\text { SDSA pass/fail } \\
\text { Fail, } \mathrm{n}(\%)\end{array}$} & \multicolumn{2}{|c|}{$\begin{array}{l}\text { On-road driving performance } \\
\text { pass/fail }\end{array}$} \\
\hline & Fail, n(\%) & Pass, n(\%) \\
\hline & $a=4(36.4)$ & $\mathrm{b}=1(09.1)$ \\
\hline Pass, n(\%) & $\mathrm{c}=0(0.0)$ & $\mathrm{d}=6(54.5)$ \\
\hline
\end{tabular}

\section{DISCUSSION}

Our stroke cohort had no apparent cognitive impairment as assessed by the MMSE, but four of seven failed the SDSA. However, seven of the PD participants with greater cognitive deficits passed the on-road evaluation. Though the small sample sizes make it difficult to tell what the results really mean, it is possible that all the seven PD participants who were current drivers had developed adequate methods to compensate for their cognitive deficits. The two PD participants who, based on performance on the SDSA, were predicted to fail but actually passed the on-road evaluation (Table 2c) also passed the simulator evaluation. Detailed data inspection revealed that the SDSA “pass" and "fail” values were quite close ( $<0.5$ difference). This finding suggests that the particpants had only very mild attention and executive function impairments for which they adequately compensated during simulated or actual driving. All four PD participants who had been predicted to fail and did fail the on-road evaluation (Table 2c) also failed the simulator evaluation (Table 2d). However, one PD particpant passed both the on-road evaluation and the SDSA assessment but failed the simulator-based evaluation. It is possible that this participant did not adjust well to the driving simulator. Yet, the accuracy (91\%) with which the outcome of the simulator evaluation predicted the outcome of the on-road evaluation is an indication of the comparabilty of the two methods. This is the first attempt, to the best of our knowledge, to adapt the SDSA for use with a US-based population. Preliminary findings showed that the adapted (US) version of the SDSA may have the potential to accurately predict driving after stroke and be a good predictor of driving with PD. Our small sample size is a major limitation of this preliminary report. As such, we caution that the predictive accuracy of the US version of the SDSA that we reported should be taken only as an indication of the potential usefulness of the SDSA to serve as a short battery to predict driving after stroke and PD. Future, larger studies are therefore needed to better assess the predictive accuracy and validity of the US version of the SDSA. Such studies should include samples of at least 60 participants (10 per SDSA measure) for each neurologic condition, and, ideally, nearly equal pass and fail rates on an on-road driving evaluation.

\section{ACKNOWLEDGMENTS}

The authors acknowledge the Augusta, GA Chapter of the National Parkinson Foundation for funding the participation of indviduals with Parkinson's disease in the study. The authors also acknowledge the contrbutions of Professor Nadina Lincoln during the adaptation of the SDSA. 


\section{REFERENCES}

Akinwuntan, A.E., Feys, H., De Weerdt, W., Pauwels, J., Baten, G., Strypstein, E. (2002). Determinants of driving after stroke. A retrospective study. Archives of Physical Medicine and Rehabilitation, 83, 334-341.

Akinwuntan, A.E., Tank, R., Vaughn, L., Wilburn, A., Easton, S. (2009). Normative Values for Driving Simulation Parameters. Conference Proceedings of the 5th International Driving Symposium on Human Factors in Driver Assessment, Training, and Vehicle Design, 161-168.

Devos, H., Akinwuntan, A.E., Nieuwboer, A., Truijen, S., De Weerdt, W. (2010). A systematic review and meta-analysis on predictors of fitness to drive after stroke. Neurology, In Press.

Fisk, G.D., Owsley, C., Pulley, L.V. (1997). Driving after stroke: driving exposure, advice and evaluations. Archives of Physical Medicine and Rehabilitation, 78, 338-345.

George, S., \& Crotty, M. (2010). Establishing criterion validity of the Useful Field of View assessment and Stroke Drivers' Screening Assessment: comparison to the results of on-road assessment. American Journal of Occupational Therapy, 64, 114-122.

Lincoln, N.B., \& Radford, K.A. (2008). Cognitive abilities as predictors of safety to drive in people with multiple sclerosis: Multiple Sclerosis, 14(1), 123-128.

Lincoln, N.B., Radford, K,A, Lee, E., Reay, A.C. (2006). The assessment of fitness to drive in people with dementia. International Journal of Geriatric Psychiatry, 21, 1044-1051.

Lundberg, C., Caneman, G., Samuelsson, S., Hakamies-Blomqvist, L., Almkvist, O. (2003). The assessment of fitness to drive after a stroke: The Nordic Stroke Driver Screening Assessment. Scandinavian Journal of Psychology, 44, 23-30.

Manual on Uniform Traffic Control Devices for Streets and Highways (2009). Washington, DC: U.S. Department of Transportation, Federal Highway Administration.

Nouri, F.M., \& Lincoln, N.B. (1992). Validation of a cognitive assessment: predicting driving performance after stroke. Clinical Rehabilitation, 6, 275-281.

Nouri, F.M., \& Lincoln, N.B. (1993). Predicting driving performance after stroke. British Medical Journal, 307, 482-483.

Radford, K.A., Lincoln, N.B., Lennox, G. (2004). The effects of cognitive abilities on driving in people with Parkinson's disease. Disability and Rehabilitation, 26(2), 65-70

Radford, K.A., Lincoln, N.B., Murray-Leslie, C. (2004). Validation of the stroke driver screening assessment for people with traumatic brain injury. Brain Injury , 18(8), 775-786.

Selander, H., Johansson, K., Lundberg, C., Falkmer, T. (2010). The Nordic stroke driver screening assessment as predictor for the outcome of an on-road test. Scandinavian Journal of Occupational Therapy, 17(1), 10-17. 